\title{
How do Dancers Want to Use Interactive Technology?
}

\author{
Appropriation and Layers of Meaning Beyond Traditional Movement Mapping \\ Raul Masu \\ ITI/LARSyS \\ Funchal, Portugal, and \\ FCT/NOVA University of Lisbon \\ Lisbon, Portugal \\ raul.masu@m-iti.org \\ Nuno N. Correia \\ University of Greenwich \\ London, UK, and \\ ITI/LARSyS \\ Funchal, Portugal \\ n.correia@greenwich.ac.uk \\ Stephan Jurgens \\ ITI/LARSyS \\ Funchal, Portugal \\ stephan.jurgens@m-iti.org
}

\author{
Ivana Druzetic \\ Hochschule Düsseldorf, \\ University of Applied Sciences \\ Düsseldorf, Germany \\ ivana.druzetic@hs-duesseldorf.de
}

\begin{abstract}
There has been an increased interest in HCI research regarding the possibilities of interactive technology applied to the field of dance performance, particularly contemporary dance. This has produced numerous strategies to capture data from the moving bodies of the dancers and to map that data into different types of display formats. In this paper, we look at the role of interactive technology in dance performance from a broader perspective, aiming at understanding the needs of dancers and their relation with the audience. To this end, we ran a focus group with ten dancers with expertise in technology. We analysed the focus group using thematic analysis. We discuss the implications for design of our results by framing the role of technology in dance performance, proposing design guidelines related to the communication to the audience, use of technology, and mapping. Moreover, we propose different levels of ambiguity and appropriation related to the creators of the performance and the audience.
\end{abstract}

\section{CCS CONCEPTS}

- Applied computing $\rightarrow$ Performing arts; • Human-centered computing $\rightarrow$ User studies; Interaction design theory, concepts and paradigms.

\section{KEYWORDS}

Dance performance, HCI, design guidelines, UCD

\section{INTRODUCTION}

In the last decades, interactive digital artefacts have become ubiquitous, and their applications have gradually evolved from workplace to everyday lives and culture. This tendency has been identified as third-wave HCI [7]. With this tendency, user experience has become central in HCI discourse [37]. It has also emerged that users tend to appropriate digital artefacts in different ways [19]. Consequently, the use and meaning of artefacts might become ambiguous, and potentially open to many interpretations [22]. In general, understanding the needs of the user has become a fundamental design activity [4], and users started to be involved in the design process.

Among the variety of contexts touched by the spread of application areas in HCI, dance performance has gained increased attention, (e.g. [11], [21], and for contemporary dance see [18]). From a computing perspective, interactive technology for dance performance can be viewed as a system including inputs (e.g. sensor technology [21]) and outputs (e.g. display strategies [23]). Related studies have analysed dance performance from a segmented perspective, mainly focusing on movement characteristics or display approaches.

Contemporary dance performance represents a complex scenario of use of interactive technology. To begin with, it is composed by two main activities: the preparation of the performance, and the performance itself; the preparation of performance is itself a development process where "choreographic material" is generated [24]. Moreover, different categories of users (dance artists: choreographers and dancers) are involved in contemporary dance performance. As depicted in the 
framework proposed by Butterworth these two main categories might play different roles [10]. Therefore, we argue that designing interactive system for dance performance requires a multifaceted approach from HCI, which takes into account the different roles of the technology in creating meaning in the overall dance performance, and the different needs of users.

In this article, we address these aspects by using early-stage co-design activity. Namely, this paper presents a focus group with ten dancers and proposes design guidelines based on the analysis of the respective results. The main objective of the focus group was to inquiring dance artists (dancers - choreographers) about a general research question: What is the role of digital systems in dance performance, in particular, interactive technology and visualization?

A secondary objective of the workshop is to understand how that role influences the dancers' communication with the audience. By this, we mean the communication from the performers to the audience, and the audience's understanding of that communication. Audience participation (communication from the audience to the performers) is out of scope for this study; it is in itself a vast topic that merits separate research. Methodologically, a novelty of this study is the involvement of users in the very initial phase of the design, inquiring possible users about the role of interactive technology in dance performance.

The rest of the paper is organized as follows: section 2 presents related works in the field of interactive technology for dance performance and on third-wave HCI; section 3 describes the focus group and section 4 the results; section 5 presents design guidelines based on the results, section 6 discusses the design implications of our findings; finally, section 7 concludes by pointing out future steps in the project and future works in general.

\section{LITERATURE REVIEW}

The literature review covers two main areas. Interactive Technology in Dance Performance discusses how digital technologies have been used as input and output in dance performances. Designing Digital Artifacts in Third-Wave HCI presents relevant concepts in HCI and related methodologies.

\subsection{Interactive Technology in Dance Performance}

The use of interactive technologies in contemporary dance, on stage, alongside dancers, already has a long and established tradition. One relevant example is the work of Mark Coniglio with MidiDancer (1989), a software that allowed a performer to control music $^{1}$. A more recent example is the work of Frieder Weiss with the system EyeCon (2004), which allows movement to control several aspects of a performance [36]. Dance artists, researchers and educators have been extensively exploring interactive digital

\footnotetext{
${ }^{1} \mathrm{http}: / /$ troikaranch.org/artistic-directors/
}

technologies in workshop and rehearsal situations [16], and in public performances and installations [26].

Different types of inputs can be used to obtain the respective dance data sets. One possible categorisation of such technology is proposed by Alaoui and colleagues [21], who distinguish among (i) positional data which are retrieved by employing motion capture systems; (ii) movement dynamics (such as acceleration/deceleration), which are recorded by means of inertial sensors; and (iii) physiological information, which can be obtained from biosignal sensor technologies.

Motion and physiological data collected through input technologies has mostly been used to obtain insights from the dancer's body, resulting in sonification [3], visualisation [23], and/or interaction with any other choreographic elements (e.g. scenic design [35], light design [8], costumes [6]). Technological artefacts, which provide the tools for such interactions, have used different mapping strategies spanning from direct mapping [36] approaches to agent-based methods [20]. Visualization strategies include visualization of moving data [5], and visualization of movement qualities [21], or more complex visualization of features extracted using different layers of analysis [11] and practitioners/artists using data art and digital performance (e.g. Choreographic Coding Labs/Motionbank ${ }^{2}$ ). To conclude, prerecorded data has been used to generate graphics synchronised with animated skeletal representations of the movements using machine learning strategies [33].

In some cases, these studies produced design suggestions or guidelines [36] and framework [11]. However, they tended to tackle specific aspects of movement or specific choreographies. With this paper we extend these works proposing high-level design guidelines for interactive technology for dance performances, addressing both input and output technology, as well as the needs of the main users (dance artists) and context of use (dance performance).

\subsection{Designing Digital Artefacts in Third-Wave HCI}

2.2.1 Appropriation and Ambiguity. Within the context of thirdwave HCI, where our study is situated, researchers started to investigate the creative uses of interactive digital artefacts [7]. In this perspective, artefacts can be used (appropriated) in ways that the designer did not expect [19] and the technology can assume multiple or adaptive meanings. Users appropriate the technology by imposing their own meaning. Dix defined appropriation as "improvisations and adaptations around technology" [17]. Aligned with this idea, Dourish proposed that to foster appropriation, design should aim to support multiple perspectives on information [19]. These multiple perspectives reflect the idea of ambiguity. Ambiguity was studied from a design perspective by Gaver, who proposed three different categories: ambiguity of information when the information is presented ambiguously; ambiguity of context, different context give to technology different meaning; ambiguity of relationship, each user has a

\footnotetext{
${ }^{2}$ http://motionbank.org/
} 
different relationship with a piece of technology. These perspectives have been prolific as design principles for artistic branches of HCI, such as music [38] and artistic installations [27]. In the area of dance, need for appropriation emerged in the design process of creative support tools for choreographers' notation [13].

2.2.2 Design of Interactive Artefacts in Third-Wave HCI. In third-wave HCI, the user increasingly becomes central in the design process of digital artefacts. User-Centered Design (UCD) is "a term to describe design processes in which end-users influence how a design takes shape" [1]. In this perspective, the design process should involve the user, in order to understand their needs in relation to the usage of the technological artifacts [4]. In addition, co-design literature underlines the increasing importance that involving users in the early stages of the design process has gained. Sanders and Stappers stressed the importance of what they call front end, formerly defined as "pre-design", a phase of the design composed of those activities that take place in order to inform the general understanding of users and contexts of use: "the goal of the explorations in the front end is to determine what is to be designed and sometimes what should not be designed and manufactured" [30]. Figure 1. represents the design phases as described by Sanders and Stappers: the "front end of the design process has been growing as designers move closer to the future users" [30].

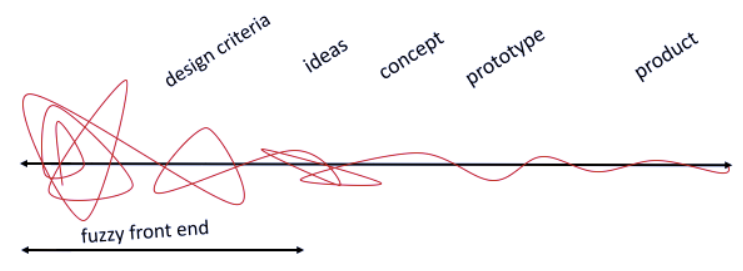

Figure 1: Design phases as proposed by Sanders [30].

Co-design approaches have been successfully used within artistic branches of third-wave HCI. For instance, UCD has been used to investigate the potential of sonic interaction in personal usage scenario [32]. Similar approaches have also been used to design specific artifacts, such as musical instruments [34], audiovisual toolkits [15], and multi-modal installations [29]. Core and colleagues [14] adopted participatory approaches to design pedagogical tools for young children that combine music technology and movement with real time algorithmic composition [28]. In the area of technology for dance, UCD has been used to design tools whose porpoise is to help the choreographic process. In particular, Ciolfi Felice and colleagues designed and developed a tablet application to support choreographers' work using animated notation $[12,13]$.

2.2.3 Co-Design and Technology for Dance Performance. Within the specific context of technology designed for dance performances, UCD is still scarcely explored, although, in recent years some projects started to involve users in the design process.
For instance, Schofield et al. [31] used participatory design workshops to involve teenagers in the design process of an artefact for dance performance. The objective of such process was to teach the participants to code: during the process the participants have learned simple coding activity while actively participating in the co-design process. Alauoi and colleagues [2] involved dancers in a participatory design in their research. Their starting point/objective was pre-defined: they aimed to design an interactive system based on movement qualities, providing visuals based on Mass-Spring Systems. Another example can be found in a project by Landry and Jeon who involved dancers in the design of an audio-visual system, but they involved only after that a first prototype was already implemented [25].

Despite being valid in defining technological specifications and producing valuable artefacts, the studies mentioned above started the design process either with a clear technology, or choreography in mind. Therefore, there is a lack of general design guidelines considering the roles of technology within the context of use: in the creation and in the performance of a dance piece.

In this paper, we address the design of technology for dance performances using UCD approach, involving the users since the earliest stage of the design process.

\section{THE STUDY}

To address our research question regarding the role of technology in dance, with the involvement of dancers, we organized a twoday co-design workshop. The workshop consisted of a series of design exercises, including a focus group. For the scope of this paper, we will present and analyse the focus group component of the workshop.

\subsection{The Focus Group}

The objective of the focus group was to gather data about the role of technology in dance, aiming to identify needs, criteria and requirements of dance practitioners. We were also interested in the role technology might have as mediator among dance artists, and between dance artists and their audience. Therefore, we structured the focus group around the following four main topics, which align with our research objectives: communication to the audience in dance performance; the role of technology in dance performance; in particular: the role of interactive technology; and the role of visuals. The purpose of this focus group was to frame the initial requirements for a future prototyping process. In this way, we followed a design perspective, informed by studies presented in the section 2.2: we started by questioning the users about their needs. The users (in this case, the dance artists) were involved from an early stage to identify needs and requirements regarding interactive and visualization technology in dance, which will be the basis for future steps in the co-design process. The focus group took place in the dance studio of Sõltumatu Tantsu Lava (STL) in Tallinn (Figure 2.) and lasted for approximately two hours. 


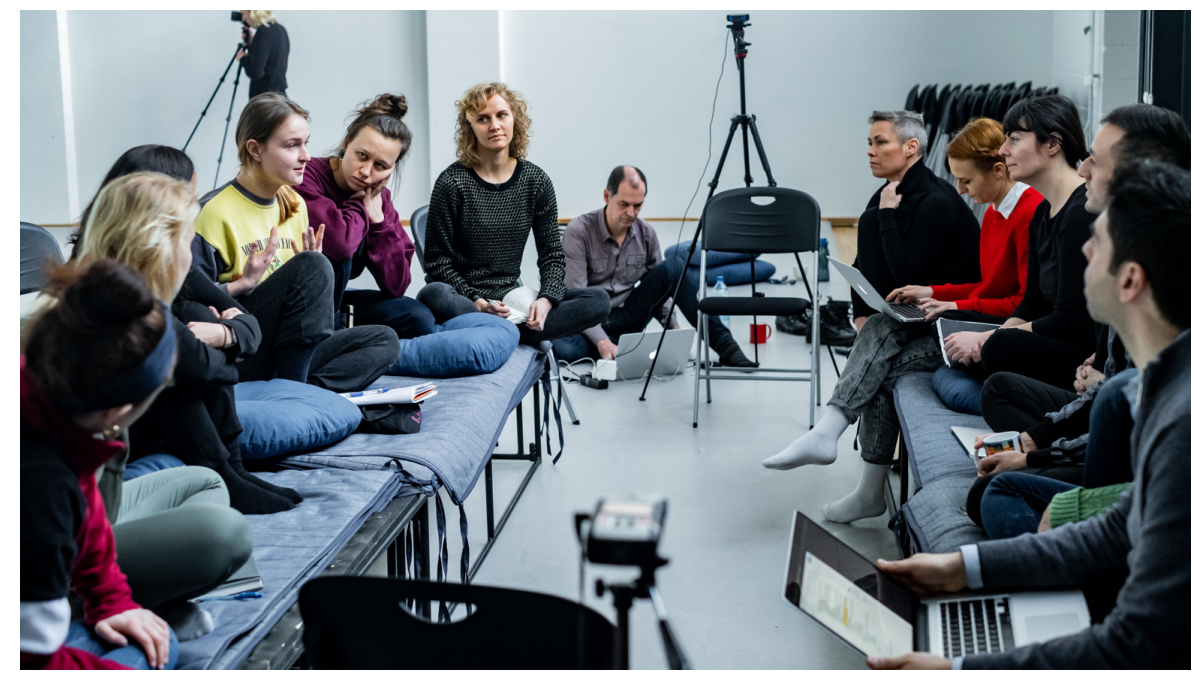

Figure 2: The setting of the focus group.

\subsection{Participants}

The participants were selected using an open call, disseminated through mailing lists related to contemporary dance. Ninety-two dancers applied to the call ( 73 female, 19 male). Each candidate was independently evaluated by six members of our team, according to i) their Curriculum Vitae as dancers, ii) previous experience with technology in dance and iii) motivation and expectations regarding the workshop. Finally, the scores were discussed and moderated. Ten dancers were selected (nine female, one male, from eight countries) and all of them participated in the study. We covered travel expenses and paid a fee for each dancer. Due to the competitive selection, all ten participants had considerable experience in contemporary dance as performers, some of them also as choreographers.

In particular, all the participants had previous experience as professionals dance artists in projects that involved technology. In most cases, our participants had experience both as dancers and as choreographers. The type of technology that our participants have used in previous works rages from VR, 3D modelling, streams of social media, different types of hardware (including Kinect and Arduino), and software (Max/MSP, openFrameworks, Processing). Referring to the different roles the dancers and choreographers have in the creative process of producing a dance piece, our participants reflect those scenarios where there is a choreographer leading the decisions. Referring to the model proposed by Butterworth, participants reflect mainly case one and two, where the choreographer is the author creating the piece [10].

\subsection{Data Collection}

We recorded audio and video and took hand-written notes during the focus-group. We transcribed and anonymised the interview data and refer to participants as P.1-P.10. The transcription has been controlled by two researchers.

\subsection{Data Analysis}

The focus group was analysed independently by two researchers using thematic analysis [9], then these analyses were crosschecked and harmonised. All the transcriptions of the focus group have been coded, the different codes have been harmonised, and finally the codes were grouped into themes. The analysis produced six themes, each with sub themes.

\section{RESULTS}

In this section, we present the results of the thematic analysis. The outcome of the analysis of the data consists of six main themes with several sub themes.

\subsection{Theme 1: Audience Characteristics}

The first theme concerns the characteristics of the audience. Our participants consider the audience to be intelligent, but also unpredictable.

- Audience is intelligent. The audience is intelligent (P.8) participants aim to make a performance for the most intelligent person in the audience (P.9).

- Audience is unpredictable. There is uncertainty about the audience: you really never know who's sitting in this audience (P.2), also the audience members may have an unexpected response (P.7).

- Audience as a close human. Finally, there is also a level of closeness with the audience - like creating this human moment of sharing something common, of human to human (P.8).

\subsection{Theme 2: Communicate with the Audience}

Related to the characteristics of the audience, several aspects concerning the communication with the audience emerged. In general, the artists agree that meaning of the experience should not be imposed to the audience. 
- Not impose one specific meaning to the audience. Relying on the fact that the audience is intelligent, the performance should not impose one specific and didactic (P.2) or prescriptive (P.3) perspective, rather create multi-layers of meaning (P.5) and information (P.9). Dancers aim at not being didactic and at not controlling the audience, even promoting provocative strategies such as deliberately causing confusion (un-focusing P.6). Dancers also do not feel the need to teach (P.2), but prefer to articulate the performance and balance the clarity, without overexposing an idea (P.3).

- Shared experience with the audience. Relying also on the notion of closeness, our participants aim at creating a sense of togetherness (P.9) with the audience. The moment of the performance has been described as a shared intimate (P.9) experience between artists and audience, together [...] and in synchrony (P.6) with the audience.

- Create safe environments for the audience. Our participants aim to create safe environments (P.5), spaces of intellectual freedom where the audience can come with their own knowledge and their own understanding (P.6).

- Considering the audience during the creation process. In order to check the clarity of my idea (P.3) some of our participants invite audience during the rehearsal asking for feedback. Our participants stressed the need of ensure clear articulation (P.3) in providing the information to the audience.

\subsection{Theme 3: Technology as Co-Shaper of the Performance}

Technology has specific characteristics, which enables the dancers to reflect on them during the creative process. In this sense, technology becomes co-shaper of the creation process: the technology is always creating some [...] setting and then it actually become a dramaturgy (P.5).

- The creative technology. The technology is creative: it's like creative dancers, there is also creative technology (P.3). This creative technology can generate creative ideas (P.2). Therefore, technology may already have a dramaturgy (P.5). There is an awareness of the duality in technological creativity: Is it a dramaturgy in the technology itself or is the choreographer that tries to use technology as a dramaturgical tool? (P.1)

- Movements fostered by the technology. A technological artifact has an impact on the movements, it imposes physical limitation (P.3) and proposes new types of technological gestures (P.2).

- The problem of excessive focus on technology. The technology should never be the focus of a performance (P.9). It should be subtle or invisible (P.9). Technology can mesmerise and fascinate (P.6) the audience, but it should not be used in this manner: there is a shared need to express something with it (P.3).

- Integration of the technology in the logic of the work. The technology should be reflected (P.3) and integrated (P.9) in the logic of the performance.
- Hacking. Our participants describe the process of using the technology as hacking the system (P.3), in a figurative sense: dancers are not using the technology the way that the technology designers meant (P.3).

\subsection{Theme 4: The Problem of Redundancy of Information}

One of the main problems that our participants identified is that technology it is often diminishing the layers of meaning in the performance.

- Technology is illustrative. Technology is too illustrative, [...] and too connected to what you are doing with movement (P.6), for this reason it risks to merely duplicate the body (P.4).

- Illustration and meaning. The visual output is too graphic, it's diminishing the multi-layered meaning (P.5). and risks to simply replicate the information (P.6).

\subsection{Theme 5: Strategies for Interaction}

From an interaction design perspective, some good practices emerged.

- Complex mappings. Unclear, divergent, or independent mappings from input to output technologies could be used to create counterpoint (P.9) between the dancers and the technology, avoiding more obvious mappings (P.6).

- Interaction Loop. Technology could create a complex mirror that challenges the movement of the body (P.9), a sort of feedback loop (P.3) that affects the choices of the dancer.

\subsection{Theme 6: Strategies for Visuals/Output Adding Layers}

This last theme clusters suggestions related to the output of the digital artefact.

- Visualize the structure. Expose the score before (P.9) or during (P.6) a performance might contribute to adding layers of meaning as it is a commentary on your own work and it's self-reflexive and it's interesting (P.6).

- Play with time-related elements. This might include displaying what things that happened in the past and [...] resonate [...] in a performance (P.9), or traces and the resonance of the movement (P.1).

- Alternative sensorial strategies. Our participants also suggested to rely on other sensorial channels, such us kinetic illustration (P.5) and sound that might be used to trigger sensation (P.10). Moreover, sound is multi-dimensional in space, and these characteristics makes sound more similar to movement as compared to visuals (P.5).

- Capture the Intelligence. Several aspects of the intelligence of a body could be captured and revealed: e.g. what's happening in the brain before the movement (P.6), record the thinking process of someone doing something incredibly complex (P.2) and also understand the intelligence of the body (P.5). 


\section{DESIGN GUIDELINES}

Our results support us to propose design guidelines for technology to be used in dance performance. Our design guidelines are organised around three high-level aspects of interactive technology for dancers:

1. Communication with the audience (from Theme 2)

(a) Technology should not impose one single perspective to the audience.

(b) Technology should contribute to create multiple layers of meaning.

2. The role of the technology in the creation of the piece (from Theme 3)

(a) Technology should provide space for appropriation, enabling the dancer to give their own use and meaning facilitate customization might be a possible strategy.

(b) Technology should be easily included in the dramaturgy of the performance - make it meaningful for the performance.

3. Input and output strategies (From Themes 4, 5, and 6)

(a) Technology should not repeat the information that the dancer is already giving with their movement (avoid overly clear mappings) (Theme 4).

(b) Technology should have a complex input-output mapping, which might be used to create a loop between technology and dancers (Theme 5).

(c) Technology should facilitate adding information contributing to multiple meanings of the performance (Theme 6). Examples that emerged in the analyses of focus group include: (i) showing non visible elements (either inner elements of the dancers or micromovements), (ii) shifting the temporal dimension of the performance (e.g. showing, in time lapses, residuals aspects of movement), (iii) showing the structure (score) of the performance.

\section{DISCUSSION}

Based on the results of the analysis of the focus-group we discuss the role of technology in contemporary dance performance; we then discuss modularity and mapping aspects of the guidelines. Finally, we discuss our finding with the lens of appropriation and ambiguity in HCI.

\subsection{The Role of Technology in Contemporary Dance Performance}

Technology plays a crucial role as co-creator of performances, but it should not be the focus. A piece of technology already has its own pre-existing dramaturgy, it imposes specific problems or limitations to the choreographer, which need to be incorporated in the ideas and meanings of the performance (Theme 3 ). In order to use technology in a meaningful way that is harmonised with the overall performance, dance artists need to appropriate the technology and give it a new meaning that is aligned with the dance piece. A performance should be composed of multiple layers of meaning (Theme 2), and technology should contribute to this multifaceted structure (Theme $4,5,6$ ). In Theme 4 , it emerged that our participants have had issues with technology when it adopts overly clear mappings, since in this case it repeats the same information of the body, creating an issue of redundancy of information. This repetition diminishes the layers of meaning of the performance. Consequently, our participants tend to dislike this characteristic of the technology, as they aim to create rich and multi-layered performances.

The need of structuring the meaning of a performance is connected to the characteristics of the audience. In Theme 1, it clearly emerged that our participants consider the audience to be intelligent. For this reason, dancers avoid having one clear meaning in the performance. On the contrary, our participants aim to have multi-layered meaning (Theme 2). We argue that technology should support this approach. Successful strategies for designing interactive technology for dancers could include either creating interactive mirror loops or by adding elements to the performance, for instance i) reveal hidden aspects of the performer, ii) play with time lapses, or iii) exposing structural elements of a performance. In general, one-to-one linear inputoutput mapping appears not to be fruitful in the context of dance performance, as it tends to visualise elements that are already clear in the movement of the body.

\subsection{Modularity and Mapping}

To achieve the principle outlined by our guidelines we propose the adoption of modular strategies in the design of interactive technology for dance performances. In particular, we propose the following points to help designers to address the proposed guidelines: (i) use different elements - building blocks - as output technology (audio or visual), these different elements should already provide functionalities that facilitate multiple usages (for instance different image processing algorithms that can be applied on the same material); and (ii) provide users with the possibility of customise mappings between the input and the output technology (for instance, giving to the choreographers the possibility to choose how to map input data on given processing algorithms).

These suggestions can help designers in applying the guidelines. Firstly, a modular structure composed of different building blocks can facilitate the choreographers to use technological artefacts during the process of creation. The modular structure facilitates different usage of the artefact itself, as each user can create different combinations of the building blocks. Therefore, each choreographer can have space to appropriate it according to their idiosyncratic characteristics.

These possibilities mentioned above can contribute to support the choreographers to include technology in the overall dramaturgy logic. Secondly, designing different functionalities for each output object reflects the guideline of complex input-output strategies. The combination of modular strategies with different functionalities and open mappings will help the user (choreographer or dancer) to employ technology to create multiple layers of meaning to propose to the audience. 


\subsection{Appropriation and Ambiguity in Interaction Design for Dance Performance}

Our participants' need for reflecting and integrating technology in the performance reverberates with the design concept of appropriation. Similarly, the need for adding layers of meaning and not imposing one single meaning in a performance resonates in the design concept of ambiguity. In Theme 3 , it emerges that the dancers' use of interactive technology implies a second creative process, whose outcome is a performance. During this process, the dancers need to appropriate the technology [17]. Moreover, the idea of layering the information is also similar to the idea of designing for appropriation proposed by Dourish: supporting multiple perspectives on information [19]. In this sense, there are two faces of appropriation: dancers appropriate the technology to create multiple layers of meaning in the performance, and the layers of meaning supports the audience to appropriate the content of the performance.

Therefore, we argue that an interactive digital artefact designed for dance performance should take into account these aspects, and not impose one restricted meaning or use, nor of meaning. On the contrary, it should support dancers to appropriate it, to embed it in the performance and contribute to the multiple layers of meaning. To this end, the artefact should already have ambiguous characteristics that facilitate the appropriation process, as advocated in [22], rather than impose one clear usage. In Theme 4, 5, and partially 6 it emerged that ambiguity can be used to build the multi-layered meaning of the performance. Therefore, adding ambiguous elements in the technology (e.g. in the rich and complex mapping possibilities from input/interaction to output/display) could enable dance artists to create those multiple layers of meaning. Based on the discussion above, we highlight two different types of ambiguity that facilitate appropriation:

- Ambiguity of use of the artifact, that facilitate the choreographer to appropriate the technology and use it in the process of creating the performance integrating it in the creative process.

- Ambiguity of mapping and meaning of the artifact, that facilitate the audience to appropriate the meaning of the performance.

\section{CONCLUSION AND FUTURE WORK}

Compared to other studies that focus on input, output, or mapping strategies in dance (e.g. [11, 21, 36]), this paper extends those studies by providing designers with a higher-level view of the role of technology in the dance performance context. We advocate that our guidelines can assist in the definition of choices and specifications for interaction design in dance. Methodologically, compared to other related studies [2, 25, 31], we adopted a UCD approach from an earlier stage, before any initial conceptual design. Thanks to this fact, our contribution addresses the role of technologies from a systemic level in the general context of a dance performance. Future works will include developing interactive prototypes based on the guidelines and evaluate them with dancers.
To conclude, in this paper we presented a focus group study on the role of interactive technology in dance performance. The participants were ten dancers with a background in dance performance with technology. The results of the focus group allowed us to frame the role of technology in dance, discuss how this contributes to the communication to the audience, analyse appropriation and ambiguity in this context, and propose design guidelines.

\section{ACKNOWLEDGMENTS}

This research is funded by 597398-CREA-1-2018-1-PT-CULTCOOP1 - Moving Digits: Augmented Dance for Engaged Audience. The first author acknowledges ARDITI - Agência Regional para o Desenvolvimento e Tecnologia under the scope of the Project M1420-09-5369-FSE-000002 - PhD Studentship. The Second author acknowledges LARSyS (UID/EEA/50009/2019). We acknowledge all participants in this research.

\section{REFERENCES}

[1] Chadia Abras, Diane Maloney-Krichmar, Jenny Preece, et al. 2004. Usercentered design. Bainbridge, W. Encyclopedia of Human-Computer Interaction. Thousand Oaks: Sage Publications 37, 4 (2004), 445-456.

[2] Sarah Fdili Alaoui, Christian Jacquemin, and Frédéric Bevilacqua. 2013 Chiseling bodies: an augmented dance performance. In CHI Extended Abstracts. 2915-2918.

[3] Paolo Alborno, Andrea Cera, Stefano Piana, Maurizio Mancini, Radoslaw Niewiadomski, Corrado Canepa, Gualtiero Volpe, and Antonio Camurri. 2016. Interactive sonification of movement qualities - a case study on fluidity. Proceedings of ISon (2016).

[4] Liam Bannon. 2011. Reimagining HCI: toward a more human-centered perspective. interactions 18, 4 (2011), 50-57.

[5] Michele Barker and Anna Munster. 2018. Moving data: artistic tendencies in visualising human and non-human movement. In Proceedings of the Conference on Electronic Visualisation and the Arts. BCS Learning \& Development Ltd., 180-187.

[6] Johannes Birringer and Michèle Danjoux. 2009. Wearable performance. Digital Creativity 20, 1-2 (2009), 95-113.

[7] Susanne Bødker. 2015. Third-wave HCI, 10 years later-participation and sharing. interactions 22, 5 (2015), 24-31.

[8] Cristiane Bouger. 2010. An interview with Gideon Obarzanek. Theatre and Performance Design: A Reader in Scenography (2010), 301.

[9] Virginia Braun and Victoria Clarke. 2006. Using thematic analysis in psychology. Qualitative research in psychology 3, 2 (2006), 77-101.

[10] Jo Butterworth. 2017. Too many cooks? A framework for dance making and devising. In Contemporary Choreography. Routledge, 115-132.

[11] Antonio Camurri, Gualtiero Volpe, Stefano Piana, Maurizio Mancini, Radoslaw Niewiadomski, Nicola Ferrari, and Corrado Canepa. 2016. The dancer in the eye: towards a multi-layered computational framework of qualities in movement. In Proceedings of the 3rd International Symposium on Movement and Computing. ACM, 6 .

[12] Marianela Ciolfi Felice, Sarah Fdili Alaoui, and Wendy E Mackay. 2016. How Do Choreographers Craft Dance?: Designing for a Choreographer-Technology Partnership. In Proceedings of the 3rd International Symposium on Movement and Computing. ACM, 20.

[13] Marianela Ciolfi Felice, Sarah Fdili Alaoui, and Wendy E Mackay. 2018. Knotation: exploring and documenting choreographic processes. In Proceedings of the 2018 CHI Conference on Human Factors in Computing Systems. ACM, 448.

[14] Cristina Core, Andrea Conci, Antonella De Angeli, Raul Masu, and Fabio Morreale. 2017. Designing a musical playground in the kindergarten. In Proceedings of the 31 st British Computer Society Human Computer Interaction Conference. BCS Learning \& Development Ltd., 41.

[15] Nuno N Correia and Atau Tanaka. 2017. AVUI: Designing a toolkit for audiovisual interfaces. In Proceedings of the 2017 CHI Conference on Human Factors in Computing Systems. ACM, 1093-1104. 
[16] Söke Dinkla and Martina Leeker. 2002. Dance and Technology. Berlin, Germany: Alexander (2002).

[17] Alan Dix. 2007. Designing for appropriation. In Proceedings of the 21st British HCI Group Annual Conference on People and Computers: HCI... but not as we know it-Volume 2. BCS Learning \& Development Ltd., 27-30.

[18] Steve Dixon. 2007. Digital performance: a history of new media in theater, dance, performance art, and installation. MIT press.

[19] Paul Dourish. 2003. The appropriation of interactive technologies: Some lessons from placeless documents. Computer Supported Cooperative Work (CSCW) 12, 4 (2003), 465-490.

[20] Marc Norman Downie and Tod Machover. 2005. Choreographing the extended agent: performance graphics for dance theater. Ph.D. Dissertation. Massachusetts Institute of Technology, School of Architecture and Planning.

[21] Sarah Fdili Alaoui, Jules Françoise, Thecla Schiphorst, Karen Studd, and Frédéric Bevilacqua. 2017. Seeing, Sensing and Recognizing Laban Movement Qualities. In Proceedings of the 2017 CHI Conference on Human Factors in Computing Systems. ACM, 4009-4020.

[22] William W. Gaver, Jacob Beaver, and Steve Benford. 2003. Ambiguity as a resource for design. In Proceedings of the SIGCHI conference on Human factors in computing systems. ACM, 233-240.

[23] Lise Amy Hansen. 2013. Making do and making new: Performative moves into interaction design. International Journal of Performance Arts and Digital Media 9, 1 (2013), 135-151.

[24] Stephan Jurgens. 2016. Three methods of designing a work ow with multimodal video annotation in interdisciplinary choreographic processes. Cambridge Scholars Publishing,159-178. UID/LIN/03213/2013;

[25] Steven Landry and Myounghoon Jeon. 2017. Participatory design research methodologies: A case study in dancer sonification. Georgia Institute of Technology

[26] Celine Latulipe, David Wilson, Sybil Huskey, Melissa Word, Arthur Carroll, Erin Carroll, Berto Gonzalez, Vikash Singh, Mike Wirth, and Danielle Lottridge. 2010. Exploring the design space in technology-augmented dance. In CHI'10 Extended Abstracts on Human Factors in Computing Systems. ACM, 2995-3000.

[27] Raul Masu, Andrea Conci, Zeno Menestrina, Fabio Morreale, and Antonella De Angeli. 2016. Beatfield: An Open-Meaning Audiovisual Exploration. In COOP 2016: Proceedings of the 12th International Conference on the Design of Cooperative Systems, 23-27 May 2016, Trento, Italy. Springer, 309-314.

[28] Raul Masu, Cristina Core, Andrea Conci, Fabio Morreale, and Antonella De Angeli. 2017. Robinflock: a polyphonic algorithmic composer for interactive scenarios with children. In Proceedings of the 14th Sound and Music Computing Conference, July 5-8, Espoo,Finland.

[29] Fabio Morreale, Raul Masu, and Antonella De Angeli. 2019. The Influence of Coauthorship in the Interpretation of Multimodal Interfaces. Wireless Communications and Mobile Computing 2019 (2019).

[30] Elizabeth B-N Sanders and Pieter Jan Stappers. 2008. Co-creation and the new landscapes of design. Co-design 4, 1 (2008), 5-18.

[31] Tom Schofield, John Vines, Tom Higham, Ed Carter, Memo Atken, and Amy Golding. 2013. Trigger shift: participatory design of an augmented theatrical performance with young people. In Proceedings of the 9th ACM Conference on Creativity \& Cognition. ACM, 203-212.

[32] Atau Tanaka, Olivier Bau, and Wendy Mackay. 2013. 11 The A20: Interactive Instrument Techniques for Sonic Design Exploration. Sonic Interaction Design (2013), 255.

[33] Ravet Thierry, Joëlle Tilmanne, Nicolasm d'Alessandro, and Sohaib Laraba 2016. Motion Data and Machine Learning: Prototyping and Evaluation. In Proceedings of the ACM CHI workshop on Human Centered Machine Learning.

[34] Luca Turchet, Andrew McPherson, and Mathieu Barthet. 2018. Co-design of a Smart Cajón. Journal of the Audio Engineering Society 66, 4 (2018), 220-230.

[35] Caitlin Vincent and Jordan Beth Vincent. 2018. Notation by Context: Digita Scenography as Artifact of Authorial Intent. Leonardo Music Journal 28 (2018), $72-76$.

[36] Robert Wechsler, Frieder Weiß, and Peter Dowling. 2004. EyeCon-A motion sensing tool for creating interactive dance, music, and video projections. In Proc. of the SSAISB Convention. Citeseer.

[37] Peter Wright, Mark Blythe, and John McCarthy. 2005. User experience and the idea of design in HCI. In International Workshop on Design, Specification, and Verification of Interactive Systems. Springer, 1-14.

[38] Victor Zappi and Andrew McPherson. 2014. Dimensionality and Appropriation in Digital Musical Instrument Design. In NIME. 455-460. 